\title{
Behandlung wider Willen
}

\author{
Winfried Rief \\ AG Klinische Psychologie und Psychotherapie, Philipps-Universität Marburg, Deutschland
}

«Die Würde des Menschen ist unantastbar»-so schön und überzeugend unsere Grundrechte formuliert sein mögen, so gibt es doch vermeintliche oder offensichtliche Ausnahmefälle, in denen man sich mit einer vollständigen Umsetzung solcher Grundrechte schwer tut. Es gibt Situationen, in denen die Priorisierung eines Grundrechts bei einer Person (z.B. autonome Entscheidungsfreiheit bezüglich medizinischer Behandlungen) entweder in Konflikt mit anderen Rechten und Pflichten oder mit den Rechten anderer Menschen steht. Im Bereich der psychiatrischen und psychotherapeutischen Behandlung betrifft dies unter anderem das Primat des Rechts auf Selbstbestimmung und Freiwilligkeit der Behandlungen. Jede Leserin und jeder Leser dieses Artikels würde wohl darauf pochen, bezüglich potenzieller Behandlungsmaßnahmen selbst entscheiden bzw. mitentscheiden zu dürfen, und das nicht erst seit Zeiten des «shared decision making» im Gesundheitswesen. Trotzdem sieht nicht nur der Gesetzgeber die Notwendigkeit, beim Recht auf Freiwilligkeit medizinischer Behandlungen auch Ausnahmen zu definieren, in denen eine Behandlung gegen den Willen der Betroffenen möglich ist (siehe Revision des $\S 1906$ des BGB vom 17.01.2013). Jedoch ist die Grenzziehung für eine solche Ausnahmeregelung höchst schwierig, und deshalb überrascht es auch nicht, dass ein Artikel aus diesem aktuellen Heft der Verhaltenstherapie [Lincoln et al., 2014], der bereits seit einigen Wochen kostenfrei auf der Homepage der Zeitschrift angerufen werden kann, bereits im Vorfeld Diskussionen ausgelöst hat.

Manche Behandlungen, die ohne Einverständnis der Betroffenen erfolgen, sind im medizinischen System durchaus undiskutiert als akzeptabel angesehen. Gibt es eine organische Ursache für einen Zustand der geistigen Verwirrung, Katatonie oder Bewusstseinseinschränkung, wird in der Regel direkt eine möglichst adäquate Behandlung eingeleitet und erwartet, dass der Betroffene nach Heilung im Nachhinein mit dieser Maßnahme einverstanden ist. Lässt sich dies jedoch auch auf den Bereich der Zwangsbehandlung bei psychischen Krankheiten übertragen? Viele der unter Zwang durchgeführten pharmakologischen Behandlungen führen nicht zu dieser positiven Post-hocBewertung der betroffenen Patienten, aber auch andere Aspek- te legen eine kritische Diskussion nahe [Lincoln et al., 2014]. Sowohl die deutsche Geschichte als auch die aktuelle Situation in autoritär geführten Ländern zeigen, dass der Behandlungsansatz in psychiatrischen Kliniken besonders gefährdet ist, politisch missbraucht zu werden, sodass unter dem Deckmantel der Notwendigkeit einer Behandlung politisch unliebsame Personen ausgeschaltet werden. Aus diesem Grund ist ein sehr vorsichtiges Abwägen, in welchen Fällen Zwangsbehandlungen möglicherweise angezeigt sind, ganz besonders wichtig.

Aber der Preis, bei der Behandlung psychischer Krankheiten das Selbstbestimmungsrecht möglichst vollständig zu erhalten, ist hoch. Personen mit unbehandelten psychischen Krankheiten haben ein erhöhtes Risiko, in die Obdachlosigkeit zu entgleiten. Jedes Jahr sterben weltweit tausende Menschen im Obdachlosenmillieu, bei denen eine psychische Erkrankung vorliegt, deren erfolgreiche Behandlung gegebenenfalls mindestens den Tod, unter Umständen auch die Obdachlosigkeit hätte verhindern können. Andere Menschen, bei denen psychische Erregungszustände auftreten und die nicht einer entsprechenden Behandlung zugeführt werden, werden in vielen Ländern kurzerhand inhaftiert - wenig verwunderlich stellt sich dabei manch einer die Frage, ob eine adäquate Behandlung hier nicht vielleicht die bessere Lösung wäre.

Neben diesen gesellschaftspolitischen Aspekten drängt sich die Notwendigkeit von Zwangsbehandlungen noch stärker im Alltag insbesondere der psychiatrischen Versorgung auf. Wer Patienten in einem massiven Erregungszustand auf psychiatrischen Stationen erlebt hat, bei denen alle Versuche zur Deeskalation hilflos verpufften, ist in aller Regel dankbar über eine gesetzliche Möglichkeit zur Behandlung gegen den Willen des Patienten, und unter Umständen noch dankbarer für den meist engagierten Einsatz der Pflegekräfte, die hier das Problem «an vorderster Front» lösen müssen. Es erscheint nicht akzeptabel, dass ein tobender Patient, der jegliche Selbstkontrolle verloren hat, Mitpatienten, Pflegekräfte, weiteres Personal, aber auch sich selbst massiv körperlich gefährdet. Auch hat die Psychiatriereform in Italien gezeigt, dass eine unkontrollierte Ablehnung solcher stationären psychiatrischen Maßnahmen diese Probleme massiv in die Familien

\section{KARGER}

Fax +497614520714

Information@Karger.com

www.karger.com (c) 2014 S. Karger GmbH, Freibur

$1016-6262 / 14 / 0241-0004 \$ 39.50 / 0$

Accessible online at:

www.karger.com/ver
Prof. Dr. Winfried Rief

AG Klinische Psychologie und Psychotherapie

Philipps-Universität Marburg

Gutenbergstraße 18, 35032 Marburg, Deutschland

rief@staff.uni-marburg.de 
verlagert, die solchen Eskalationssituationen unter Umständen noch hilfloser ausgeliefert sind.

Trotz dieser nachvollziehbaren Aspekte, die für Zwangsbehandlungen sprechen, bleibt die Tatsache bestehen, dass es sich um eine Menschenrechtsverletzung handelt, und diese deshalb bestenfalls einzelnen Ausnahmesituationen vorbehalten bleiben sollte. Der Beitrag von Lincoln und Kollegen in diesem Heft tendiert zu dem Fazit, dass manches dagegen spricht, die Möglichkeiten für Zwangsmaßnahmen gesetzlich zu liberalisieren: je weniger Ausnahmen der Gesetzgeber erlaubt, desto eher werden nach Ansicht der Autorinnen auch (gegebenenfalls bessere?) Alternativstrategien zur Prävention und zum Umgang mit solchen Ausnahmesituationen entwickelt. Eine solche Schlussfolgerung kann jedoch kaum ausreichend wissenschaftlich belegt werden, da Forschung in diesem Bereich schwierig ist, noch dazu wenn es sich um die Auswirkungen gesetzlicher Regelungen in unterschiedlichen Ländern handelt. Der Vergleich der Effekte bei unterschiedlichen Ländern kann bestenfalls korrelative Überlegungen begründen, jedoch keine eindeutigen Schlussfolgerungen zulassen. So wird in den USA das Recht auf Selbstbestimmung von Patienten enorm hoch gehalten; auf der anderen Seite gibt es hier aber auch ein Heer an (unbehandelten) Obdachlosen mit psychischen Krankheiten; die Gefängnisse sind gefüllt mit psychisch Kranken, für die zum Teil sogar die Todesstrafe in Erwägung gezogen wird. Gleichzeitig gibt es aber auch Länder wie Neuseeland, die bei psychischen Erkrankungen zwar ebenfalls kaum Möglichkeiten zur Zwangsbehandlung vorsehen, in denen jedoch die entsprechenden Patienten mit psychischen Krankheiten deutlich weniger auf der Straße oder im Gefängnis landen. Letztendlich bleibt trotz der genannten Vergleiche die Hauptfrage bestehen, die für alle Länder gilt: Wird alles Menschenmögliche in der Gesellschaft getan, um die Verletzung dieses Rechts auf Selbstbestimmung auf ein Minimum zu begrenzen?

In den letzten 30 Jahren gab es eine deutliche Verbesserung in der Schulung von Mitarbeitern der Psychiatrie zum Einsatz von Deeskalationsstrategien in Ausnahmezuständen. Auch wenn dies z.B. in Deutschland noch nicht flächendeckend umgesetzt wird, sind diese grundsätzlichen Initiativen als ausgesprochen positiv zu bewerten. Trotzdem stellt sich auch die Frage, ob bereits genug getan wird, oder ob es sich nicht um einen kontinuierlichen Verbesserungsprozess handeln muss. Zwar gibt es gerade aus der sozialpsychologischen Forschung einige Ansätze, wie Deeskalation wissenschaftlich fundiert erreicht werden kann, jedoch sind auch die wissenschaftlichen Bemühungen in diesem Feld als nicht ausreichend zu bewerten. Somit ist die Forschung gefordert, diesbezüglich weitere Verbesserungsvorschläge zu entwickeln, die dann in den entsprechenden Schulungsmaßnahmen für die Mitarbeiter umgesetzt werden können.

Für viele betroffene Patienten ist allein die Zwangseinweisung in die psychiatrische Klinik sowie der Aufenthalt an diesem fremden und für manche feindselig wirkenden Ort eine Provokation, die die Wahrscheinlichkeit von psychischen Aus- nahmezuständen deutlich erhöht. Deshalb stellt sich auch die Frage, ob genügend ambulante Versorgungsmöglichkeiten für Personen bestehen, die bezüglich der Entwicklung solcher Ausnahmezustände als Risikopersonen bezeichnet werden müssen. Die alte Faustregel «Der psychisch Schwerkranke kommt in die Psychiatrie, der weniger Schwerkranke wird ambulant versorgt» entbehrt bei genauer Betrachtung einer gesicherten Grundlage. Gerade bei Patienten, die zu solchen Ausnahmezuständen neigen, ist denkbar, dass eine zwar intensive, aber in der üblichen Umgebung stattfindende Behandlung deutlich mehr Erfolge bringt, anstatt den Patienten gegen seinen Willen auf eine ortsfremde geschlossene Station zu bringen. Allerdings ist auch offensichtlich, dass eine solche wohnortorientierte Behandlung hohen Personaleinsatz fordert, was nicht nur mit erhöhten Personalkosten per se, sondern auch mit zusätzlichen Schulungsmaßnahmen einhergehen würde.

Diverse weitere Strategien zur Deeskalation sind bereits ausformuliert worden, werden jedoch nur unbefriedigend umgesetzt. Hierzu zählt z.B. der systematische Einsatz der Angehörigen, die bei den meisten Betroffenen eher zur Beruhigung beitragen können und damit Eskalationsprozesse hemmen.

Nicht zuletzt soll auf die hohe Bedeutung auch baulicher Setting-Variablen hingewiesen werden [Linden, 2010]. Hierzu sei eine kleine Anekdote aus der Biografie des Mitherausgebers der Verhaltenstherapie eingestreut : «Als junger Psychologe war ich auf einer Station tätig, Altbau, Bettensäle mit maximal 12 Patienten pro Zimmer, langen Fluren, etc. Inadäquate Verhaltensweisen wie Einnässen, Einkoten, aggressive Ausbrüche waren an der Tagesordnung. Mit der kompletten Station wurde in einen Neubau umgezogen, maximal 3 Patienten pro Zimmer, freundliche Umgebung. Die Häufigkeit der zuvor beschriebenen inadäquaten Verhaltensweisen reduzierte sich auf einen kleinen Bruchteil, trotz gleicher Patientenstichprobe.» Dieses Beispiel soll unterstreichen: Auch über bauliche Setting-Variablen können Maßnahmen zur Deeskalation erreicht werden. Auch dies kostet Geld, jedoch ist der Schutz der Bürgerrechte und Menschenwürde ein Gut, für das sich Investitionen lohnen.

Die Ärztin und der Arzt, die in solchen schwierigen Situationen der Eskalation Entscheidungen treffen müssen, haben einen berechtigten Anspruch auf Rechtssicherheit. Daneben sind jedoch alle beteiligten Expertengruppen aufgerufen, weitere Verbesserungsmöglichkeiten zur Prävention solcher Eskalationssituationen sowie zur Deeskalation in den entsprechenden Krisensituationen kontinuierlich weiterzuentwickeln.

\section{Literatur}

Lincoln TM, Heumann K, Teichert M: Das letzte Mittel? Ein Überblick über die politische Diskussion und den Forschungsstand zum Einsatz medikamentöser Zwangsbehandlung in der Psychiatrie. Verhaltenstherapie 2014;24:22-32.

Linden M: Therapeutisches Milieu. Healing Environment in medizinischer Rehabilitation und stationärer Behandlung. Berlin, MMV, 2010. 